\title{
Frequency combs with weakly lasing exciton-polariton condensates
}

\author{
K. Rayanov, ${ }^{1}$ B. L. Altshuler, ${ }^{1,2}$ Y. G. Rubo, ${ }^{3}$ and S. Flach ${ }^{1,4}$ \\ ${ }^{1}$ New Zealand Institute for Advanced Study, Centre for Theoretical Chemistry \& Physics, \\ Massey University, 0745 Auckland, New Zealand \\ ${ }^{2}$ Physics Department, Columbia University, New York, USA \\ ${ }^{3}$ Instituto de Energías Renovables, Universidad Nacional Autónoma de México, Temixco, Morelos, 62580, Mexico \\ ${ }^{4}$ Center for Theoretial Physics of Complex Systems, Institute for Basic Science, Daejeon, Korea
}

(Dated: June 23, 2022)

\begin{abstract}
We predict the spontaneous modulated emission from a pair of exciton-polariton condensates due to coherent (Josephson) and dissipative coupling. We show that strong polariton-polariton interaction generates complex dynamics in the weak-lasing domain way beyond Hopf bifurcations. As a result, the exciton-polariton condensates exhibit self-induced oscillations and emit an equidistant frequency comb light spectrum. A plethora of possible emission spectra with asymmetric peak distributions appears due to spontaneously broken time-reversal symmetry. The lasing dynamics is affected by the shot noise arising from the influx of polaritons. That results in a complex inhomogeneous line broadening.
\end{abstract}

PACS numbers: 42.55.Ah, 78.67.-n, 71.36.+c, 42.79.Hp

Condensation of exciton-polaritons (EP's) in semiconductor microcavities formed by two distributed Bragg mirrors with quantum wells between them has been experimentally observed [1 5]. Being incoherently excited in the microcavity, EP condensates are in general out of thermodynamic equilibrium. EP condensates refuel their particle depot through absorption of cavity photons and emit coherent light due to tunneling of the composite EP states through distributed Bragg mirrors. Sample inhomogeneity, either accidental or intentional, can induce several condensation centers (CC's) $3,6,8$. At low enough pumping, one expects a system of disconnected BEC droplets emitting light at different uncorrelated frequencies. As the pumping increases the condensates tend to establish mutual coherence and emit in a laser mode 6]. Already two CCs can synchronize and emit at a single joint frequency [9, 10. This is possible because the condensates exchange particles due to Josephson coupling and adjust their emission frequencies, which in turn depend on the number of condensed particles due to the polariton-polariton repulsion. In addition to the coherent Josephson coupling, there can be a dissipative (radiative) coupling between CC's, which reflects the dependence of the losses in the system on the symmetry of singe-particle states. That new stationary regime called weak lasing takes place when pumping rates reside between some minimal and maximal rates of losses [10. In the weak lasing regime, the system is stabilized by the formation of specific many-particle states which adjust the balance between gain and loss in the system.

In this Letter we show that in the weak lasing regime two CCs can emit not only at a single frequency, but also at a whole frequency comb which in principle contains an infinite number of equidistant lines of coherent lase-like radiation. This emission reflects the fact of formation of spontaneous selfsustained anharmonic oscilla- tions of both the occupation numbers and the relative phase between the condensates, in sharp contrast to previously reported damped Josephson oscillations [11 13. We study possible emission spectra and the way they are affected by noise. While the emission frequency of singleline EP lasers resides in the eV range [1, 3, 6, 7, 14, the modulation frequency of comb emission can be adjusted to be in the terahertz and sub-terahertz range. Filtering out of the high-frequency component through optical demodulation yields the low-frequency coherent signal as a new promising type of coherent terahertz emitters. The EP self-induced oscillation is also a novel mechanism of optical frequency comb generation as compared to modelocked lasers [15, 16] and optical microresonators [17, 18].

Consider two coupled EP condensates with order parameters

$$
\psi_{1,2}=\sqrt{n_{1,2}} e^{i(\Phi \mp \phi)},
$$

where $n_{1,2}$ are the occupations of the two condensates, $\Phi$ is the total phase and $2 \phi$ is the phase difference. The time evolution of $\psi_{1,2}$ is governed by the Langevin equations $(\hbar=1)$ 10

$$
\begin{aligned}
\frac{d \psi_{\mu}}{d t}=- & \frac{1}{2}\left(g \psi_{\mu}+\gamma \psi_{\nu}\right) \\
& -\frac{\mathrm{i}}{2}\left(2 \omega_{\mu} \psi_{\mu}-J \psi_{\nu}+\alpha\left|\psi_{\mu}\right|^{2} \psi_{\mu}\right)+f_{\mu}(t),
\end{aligned}
$$

where $\mu \neq \nu=1,2$ label the condensates . The parameter $g=\Gamma-W$ describes the difference between the rates of losses $\Gamma$ and pumping $W, \omega_{\mu}$ denote the singeparticle energies of the condensates, the parameters $\gamma$ and $J$ define dissipative and coherent coupling between the condensates, respectively, and $\alpha$ is the polaritonpolariton interaction constant. The last term in Eq. (2) is the Gaussian white noise satisfying $\left\langle f_{\mu}(t) f_{\mu^{\prime}}\right\rangle=0$ and $\left\langle f_{\mu}(t) f_{\mu^{\prime}}^{*}\left(t^{\prime}\right)\right\rangle=W_{\mu} \delta_{\mu \mu^{\prime}} \delta\left(t-t^{\prime}\right)$. Due to gauge invariance, 
only the frequency detuning $\omega$ is relevant and in what follows we will count the frequency from $\omega_{0}=\left(\omega_{1}+\omega_{2}\right) / 2$. Rescaling time we can fix $\gamma=1$ and, since rescaling the condensate amplitudes is equivalent to a change of $\alpha$, we can set $\alpha=2$ without loss of generality.

The dissipative coupling induces a relative phase $\varphi$ dependent dissipation in the system. This can be observed from the eigenvalues $\lambda$ which control the condensate evolution $\psi_{1,2} \sim \mathrm{e}^{\lambda t}$ in the absence of interaction [10]. For sufficiently large $\gamma / g$ one of the eigenmodes turns unstable. Therefore, the dissipative coupling acts as a phaseselective pump which depends on the relative phase $\varphi$ : it pumps one eigenmode while keeping the other one lossy. In this regime nontrivial weak lasing states are formed (see Ref. [10] for a complete account).

First we consider the noise-free case $\left(f_{1,2}=0\right)$ in order to determine the attractors of the system (2). Two nontrivial solutions $\mathrm{F}^{ \pm}$to Eqs. (2) were identified in Ref. [10. They are characterized by nonzero timeindependent triplets $\left\{n_{1}, n_{2}, \varphi\right\}_{ \pm}$with the total occupation $n=n_{1}+n_{2}=\left[\left(g^{2}+J^{2}\right) R^{ \pm}+g \omega\right] / g J R^{ \pm}, n_{1}=n(1-$ $\left.J R^{ \pm}\right) / 2, n_{2}=n\left(1+J R^{ \pm}\right) / 2, \phi=\left(\pi-\arctan R^{ \pm}\right) / 2$, where $R^{ \pm}= \pm \sqrt{\left(1-g^{2}\right) /\left(g^{2}+J^{2}\right)}$. The total phase $\Phi$ of the condensates satisfies

$$
\dot{\Phi}=\left(-\frac{1}{2}+\frac{J \cos (2 \phi)}{4 \sqrt{n_{1} n_{2}}}\right)\left(n_{1}+n_{2}\right)+\frac{\sin (2 \phi)}{4 \sqrt{n_{1} n_{2}}}\left(n_{1}-n_{2}\right),
$$

and the rhs in (3) gives a time-independent frequency $\dot{\Phi}=-\Omega_{0}$ for these solutions. The two centers evolve in a coherent fashion $\psi_{1,2} \sim \mathrm{e}^{-i \Omega_{0} t}$ and $\Omega_{0}$ defines the blue-shift of the emission line with respect to the average single-particle frequency. In the subspace $\left\{n_{1}, n_{2}, \phi\right\}_{ \pm}$ these states correspond to fixed points [19. In parts of the control parameter space these states are stable, and should manifest themselves as weak lasing states. The $F^{ \pm}$states loose stability at $g=g_{c}^{ \pm}[10$

$$
2\left(g_{c}^{4}+J^{2}\right) R^{ \pm}=\left(g_{c}^{2}+1\right)\left[\omega g_{c}+\left(g_{c}^{2}+J^{2}\right) R^{ \pm}\right]
$$

We plot the two instability curves in the $\{g, \omega\}$ space at fixed $J=0.1$ in Fig. 1 (solid lines). The $F^{ \pm}$states are unstable in the shaded areas $\mathrm{LC}^{ \pm}$. In particular they are both unstable in the joint area $\mathrm{LC}^{+}$and $\mathrm{LC}^{-}$, where the trivial solution $n_{1}=n_{2}=0$ is unstable as well. What are then the stable stationary states of the system, if any?

The answer is obtained by linearizing the phase space flow around $F^{ \pm}$in the subspace $\left\{n_{1}, n_{2}, \phi\right\}_{ \pm}$. At $g=$ $g_{c}^{ \pm}$two corresponding eigenvalues are purely imaginary $\pm i \Delta \Omega$, with their real parts changing sign. As a result, a supercritical Hopf bifurcation occurs, where stable limit cycles $\mathrm{LC}^{ \pm}$with frequency

$$
\Delta \Omega=\sqrt{2 g^{2}+J^{2} / g^{2}+J^{2}+\omega g / R^{ \pm}}
$$

are born around the respective unstable fixed points $\mathrm{F}^{ \pm}$ [20, 21].

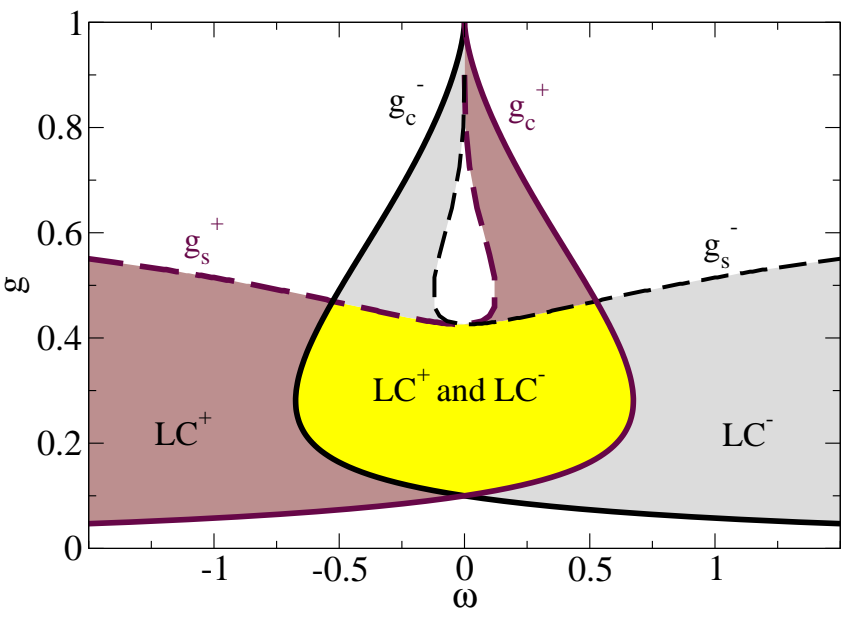

FIG. 1: (Color online) Limit cycles $\mathrm{LC}^{ \pm}$appear in the shaded areas in the $\omega, g$-parameter space. Stable $\mathrm{LC}^{ \pm}$are born through Hopf bifurcations at the solid $g_{c}^{ \pm}$line and turn unstable at the dashed $g_{s}^{ \pm}$lines where they undergo period doubling bifurcations. They are the only stable attractors to coexist in the central (yellow) region. Here $J=0.1 \gamma$.

Away from the bifurcation line the LCs increase the oscillation amplitudes, deform, and change their frequency. The coexistence region of $\mathrm{LC}^{+}$and $\mathrm{LC}^{-}$grows in size as the Josephson tunneling is reduced. At the Hopf bifurcation, where a LC emerges, $n_{1,2}, \varphi$ and also $\dot{\Phi}$ become periodic functions of time with period $T=2 \pi / \Delta \Omega$. Then they may be expanded in a Fourier series with frequency harmonics $N \Delta \Omega$ and $N=0, \pm 1, \pm 2, \ldots$ The integration of the constant term $(N=0)$ in the Fourier series of $\dot{\Phi}$ results in a linear time dependence, $\Phi_{D C}=-\Omega_{0} t$, similar to $F^{ \pm}$. Therefore

$$
\psi_{\mu}(t)=p_{\mu}(t) e^{-i \Omega_{0} t}
$$

where the functions $p_{\mu}(t)=p_{\mu}(t+T)$ are periodic in time. The Fourier spectrum of $\psi_{\mu}(t)$ is equidistant with frequency harmonics positioned at $\Omega_{0}+N \Delta \Omega$.

Approaching the dashed lines $g_{s}^{ \pm}$in Fig 1 the corresponding LC turns unstable and undergoes a period doubling bifurcation. This gives rise to a new stable period-doubled LC, which however again quickly undergoes a period doubling bifurcation. A period doubling route to chaos along a Feigenbaum scenario leads to chaotic attractors [21]. Therefore just two coupled exciton-polariton condensates suffice to produce an extremely rich and complex synchronized dynamics.

Experimentally the polariton order parameter is detected by analyzing the emitted light from the microcavity. In near-field measurements, only small parts of the sample, like one condensation center, can be probed. Our aim is to calculate the spectral density $I_{1,2}(\Omega)$ of the radiation corresponding to different nontrivial attractors. Applying a Fourier transformation (FT) we have

$$
I_{\mu}(\Omega)=\left|\mathrm{FT}\left(z_{\mu}(t)\right)\right|^{2}, \quad \mu=1,2 .
$$



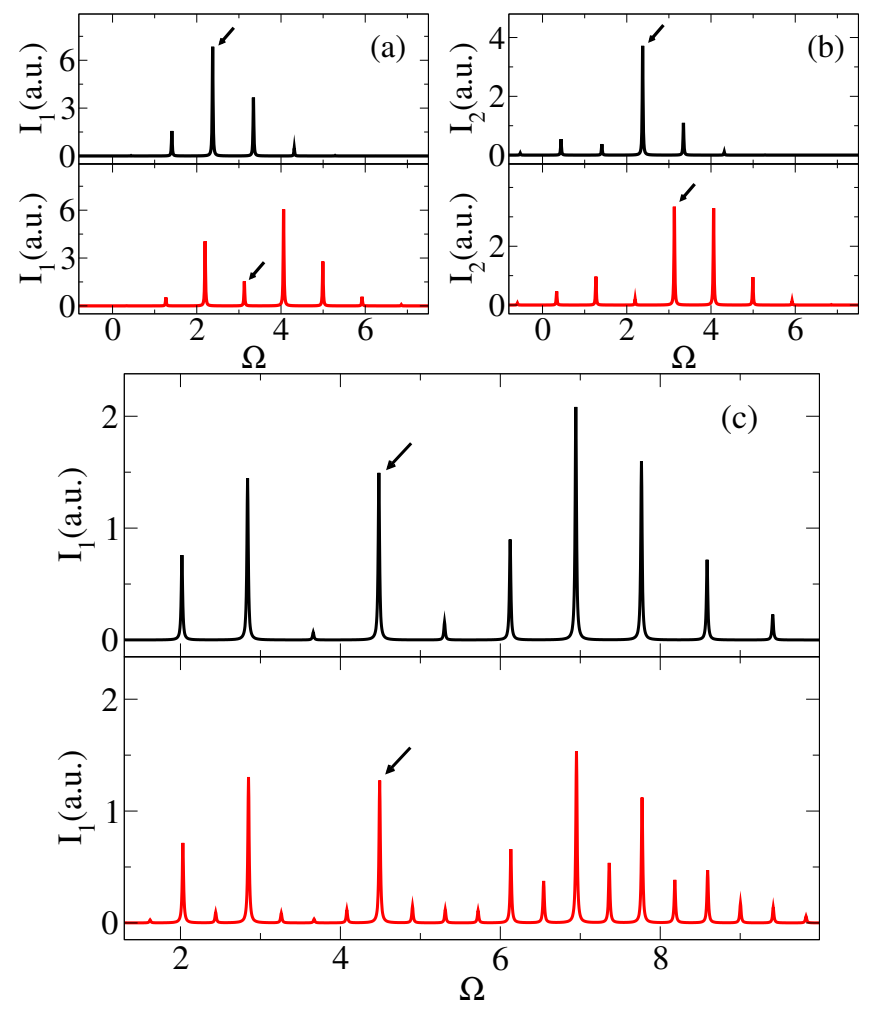

FIG. 2: (Color online) Asymmetric frequency combs in the near-field spectrum from a $\mathrm{LC}^{+}$(a),(b) and a period doubled $\mathrm{LC}^{+}$(c). Here $\omega=0, J=0.1$. The small arrows indicate the position of the respective $\mathrm{N}=0$ peak. (a) $I_{1}$ with $g=0.19$ (upper panel), $g=0.27$ (lower panel). (b) The same as (a) but for $I_{2}$. (c) $I_{1}$ for $g=0.425$ (upper panel) and $g=0.426$ (lower panel, period doubled LC spectrum). For visualization, a small artificial Lorentzian line width was added to all emission lines.

In the fixed points $F^{ \pm} \dot{n}_{1,2}=\dot{\phi}=0$, and the time dependence comes from the evolution of the total phase $\Phi=-\Omega_{0} t$

$$
\psi_{\mu}(t)=C_{\mu} e^{-i \Omega_{0} t},
$$

with constants $C_{\mu}$. Thus the condensates emit light at frequency $\Omega_{0}$ fully synchronized. The emission spectrum consists of only one peak, in contrast to the case of noninteracting polaritons, where two separated peaks are expected.

Since the limit cycles $\mathrm{LC}^{ \pm}$are characterized by an equidistant spectrum, we first numerically compute the corresponding frequency positions, and then calculate the intensity of each frequency harmonics using a Fourier series expansion. The resulting spectra are shown in Fig $2(a, b)$. Close to the Hopf bifurcation, there is only one considerable emission peak originating from the $F^{+}$ spectral line (Fig2(a,b) upper panels). Further away from the Hopf bifurcation, the satellite peaks grow to form a frequency comb with asymmetric tails (Fig $2(a, b)$ lower panels). The comb also acquires several peak max- ima, with the highest peak originating from a satellite with nonzero $N=2$ (Fig 2(a,b) lower panels). When the LC undergoes a period doubling bifurcation, the comb becomes twice as dense (Fig/2(c)).

The typical modulation frequency is independent of the polariton-polariton interaction constant $\alpha$ and is of the order of the coupling constant $\Delta \Omega \sim \gamma$. For typical dissipative rates in semiconductor microcavities $\Gamma \sim 10^{12} \mathrm{ps}^{-1}$ one expects condensate pairs with dissipative coupling $\gamma \sim 5 \times 10^{11} \mathrm{ps}^{-1}$ and below. This would permit to generate frequency combs with terahertz separation between the individual peaks. Additional reduction of this separation by period doubling can shift the modulation frequency into the millimeter range.

Finally, we consider the influence of noise in Eq. (2). In general, it will broaden the peaks discussed so far, and can lead to a merging of peaks with small enough spacing. The emission spectrum can be obtained using the Wiener-Khinchin theorem,

$$
I_{\mu}(\Omega)=\frac{1}{\pi} \Re \int_{0}^{\infty}\left\langle\psi_{\mu}(t) \psi_{\mu}^{*}(0)\right\rangle e^{i \Omega t} d t
$$

where $\left\langle\psi_{\mu}(t) \psi_{\mu}^{*}(0)\right\rangle$ is the auto-correlation of the, now, random process $\psi_{\mu}(t)$.

The $F^{ \pm}$states are periodic orbits in the full fourdimensional phase space, and the dynamics along these periodic orbits is parameterized by the total phase $\Phi$. While fluctuations off the periodic orbit will relax back, fluctuations along the orbit do not, and will enforce diffusion of $\Phi$ on the orbit. The latter fluctuations can be shown to form a Lorentzian line with the full width at half maximum (FWHM) given by $W\left(n_{1}+n_{2}\right) / 8 n_{1} n_{2}$ 22. Note that the FWHM is inversely proportional to the number of particles in the condensate, as it should be for a laser.

In contrast to the $F^{ \pm}$states, the $\mathrm{LC}^{ \pm}$states are formed by the motion on a torus in the full phase space. The stability of the attractor demands that fluctuations off the torus relax back. Fluctuations along the torus surface enforce a diffusion on it. The two nontrivial phases which diffuse, are the total phase $\Phi$ and the second phase angle, which characterizes the position on the limit cycle.

Close to the Hopf bifurcation, and in the presence of only a few satellite peaks, we can obtain a closed formula for the line width. To parameterize the $\mathrm{LC}$ we introduce two time arguments: one originating from the total phase and the other from the LC phase. Noise in these time arguments, according to Eq. (6), leads to

$$
\begin{aligned}
\psi_{\mu}(t) & =g_{\mu}\left(t+\frac{1}{-v(t)} \int P(\tau) d \tau\right) e^{-i \Omega_{0} t+i \int F(\tau) d \tau} \\
& =\sum_{N} C_{\mu}^{N} e^{-i N \Delta\left(t+\frac{1}{-v(t)} \int P(\tau) d \tau\right)} e^{-i \Omega_{0} t} e^{i \int F(\tau) d \tau},
\end{aligned}
$$


where the periodic function $p(t)$ has been expanded in a Fourier series with coefficients $C^{N}$ and $v(t)$ is the velocity of the noise-free trajectory along the LC in the three-dimensional space $\left\{n_{1}, n_{2}, \varphi\right\}$. The noise term $P(t)$ is the projected noise along the LC, while $F(t)=$ $(1 / 4 \mathrm{i})\left[\sum_{\mu}\left(f_{\mu}(t) / \psi_{\mu}\right)-\right.$ c.c. $]$ is the noise added to the rhs of Eq. (3) for $\dot{\Phi}$. Note that perturbing the time argument of $p(t)$ already accounts for a part of the noise $F(t)$, because $\Phi(t)$ is time periodic. However close to the Hopf bifurcation this periodic part is negligible compared to the DC part of $\Phi(t)$, so that the above separation of noise in the time arguments is justified.

The choice of the two time arguments is convenient when characterizing the dynamics of the noise-free LC. In the presence of noise however it leads to nonzero correlations

$$
\left\langle(N P(t)+F(t))\left(N P\left(t^{\prime}\right)+F\left(t^{\prime}\right)\right)\right\rangle=2 \kappa_{N}(t) \delta\left(t-t^{\prime}\right) .
$$

Denoting $\left\langle P(t) P\left(t^{\prime}\right)\right\rangle=\kappa_{P P} \delta\left(t-t^{\prime}\right),\left\langle F(t) F\left(t^{\prime}\right)\right\rangle=$ $\kappa_{F F} \delta\left(t-t^{\prime}\right),\left\langle P(t) F\left(t^{\prime}\right)\right\rangle=\kappa_{P F} \delta\left(t-t^{\prime}\right)$, we have

$$
\kappa_{N}=\kappa_{F F}+2 N \kappa_{P F}+N^{2} \kappa_{P P}
$$

with an asymmetric dependence of $\kappa_{N}$ on $N$.

The intensity of the noise $\kappa_{N}(t)$ is periodic in time, which originates from the oscillation of occupation numbers and relative phase for evolution along the LC. Experimentally, the measurement time spans many LC periods and one can use the average value $\bar{\kappa}_{N}=$ $\frac{1}{T} \int_{0}^{T} \kappa_{N}(\tau) d \tau$. Then

$$
I_{\mu}(\Omega)=\frac{1}{\pi T} \sum_{N}\left|C_{\mu}^{N}\right|^{2} \frac{\bar{\kappa}_{N}}{\bar{\kappa}_{N}^{2}+\left(\Omega-\Omega_{0}-N \Delta \Omega\right)^{2}} .
$$

We obtain a Lorentzian for every emission line, with an $N$-dependent width $\bar{\kappa}_{N}$ according to Eq. 111). The $N$-dependence of the line broadening $\bar{\kappa}_{N}$ follows from Eq. 12 and shows two remarkable features. First, there is an $N$-symmetric line broadening with increasing $N$ which is very strong and proportional to $N^{2}$. Second, there is an asymmetric contribution $\sim N$ which originates form nonzero correlations $\kappa_{P F}$. It may lead to a satellite peak becoming more narrow than the main peak $N=0$, and can further enhance the asymmetry of the spectrum, as compared to the noise-free case.

To calculate the line width $\bar{\kappa}$ numerically, we denote by $(A(t), B(t), C(t))^{\mathrm{T}}$ the normalized tangent vector along the LC in the coordinates $\left(\phi, n_{1}, n_{2}\right)^{\mathrm{T}}$. Then $P$ is the noise in $\dot{\phi}, \dot{n}_{1}, \dot{n}_{2}$ projected onto this tangent and we can evaluate from Eq. 11.

$$
\begin{aligned}
\kappa_{N}= & \frac{W_{1}}{n_{1}}\left(N^{2}\left(\frac{A^{2}}{4}+B^{2} n_{1}^{2}\right)-\frac{N A}{4}+\frac{1}{16}\right) \\
& +\frac{W_{2}}{n_{2}}\left(N^{2}\left(\frac{A^{2}}{4}+C^{2} n_{2}^{2}\right)+\frac{N A}{4}+\frac{1}{16}\right) .
\end{aligned}
$$
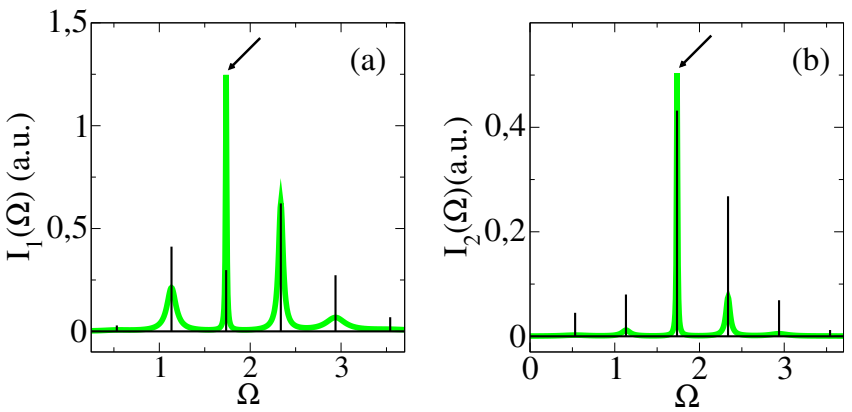

FIG. 3: (Color online) Noisy (green lines) against noise-free (black frequency combs) spectrum of a $\mathrm{LC}^{+}$at $g=0.7, \omega=$ $0, J=0.5$, for (a) condensation center 1 and (b) condensation center 2. The arrows indicate the central peak. Here $W=$ 0.02 .

We show the spectrum with inhomogeneous line broadening compared to the noise-free case in Fig 3 . Due to noise, the asymmetry of the spectrum is enhanced and the strict equidistance of emission lines is relaxed for strong enough line broadening. We note that this shape together with the equidistance of emission peaks is very reminiscent of experimentally obtained spectra in [7.

Dissipative coupling between coexisting excitonpolariton condensates in semiconductor microcavities together with strong polariton-polariton repulsion leads to a rich dissipative nonlinear dynamics already for two coupled condensates. We showed that, in addition to full synchronization [9, 10, formation of limit cycles gives rise to frequency combs of equidistant asymmetric spectral lines. The frequency offset and line spacing of the combs are tunable through the control parameters. Through period doubling, the line spacing can be additionally reduced by an order of magnitude. This modulated emission can be useful for terahertz and sub-terahertz applications. Shot noise from the pump results in a complex diffusion in phase space and has strong impact on higher order satellite peaks.

Acknowledgments.-We thank I. Aleiner, and A. Kavokin for valuable discussions. YGR acknowledges support from the EU FP7 IRSES Project POLAPHEN.

[1] J. Kasprzak, M. Richard, S. Kundermann, A. Baas, P. Jeanbrun, J. M. J. Keeling, F. M. Marchetti, M. H. Szymańska, R. André, J. L. Staehli, V. Savona, P. B. Littlewood, B. Deveaud, and Le Si Dang, Nature 443, 409 (2006).

[2] R. Balili, V. Hartwell, D. Snoke, L. Pfeiffer, and K. West, Science 316, 1007 (2007).

[3] C. W. Lai, N. Y. Kim, S. Utsunomiya, G. Roumpos, H. Deng, M. D. Fraser, T. Byrnes, P. Recher, N. Kumada, T. Fujisawa, Y. Yamamoto, Nature 450, 526 (2007).

[4] J. J. Baumberg, A. V. Kavokin, S. Christopoulos, A. J. D. Grundy, R. Butté, G. Christmann, D. D. Solnyshkov, 
G. Malpuech, G. Baldassarri Höger von Högersthal, E. Feltin, J.-F. Carlin, and N. Grandjean, Phys. Rev. Lett. 101, 136409 (2008).

[5] E. Wertz, L. Ferrier, D. D. Solnyshkov, P. Senellart, D. Bajoni, A. Miard, A. Lemaître, G. Malpuech, and J. Bloch, Appl. Phys. Lett. 95, 051108 (2009).

[6] A. Baas, K. G. Lagoudakis, M. Richard, R. André, Le Si Dang, and B. Deveaud-Plédran, Phys. Rev. Lett. 100, 170401 (2008).

[7] D. N. Krizhanovskii, K. G. Lagoudakis, M. Wouters, B. Pietka, R. A. Bradley, K. Guda, D. M. Whittaker, M. S. Skolnick, B. Deveaud-Plédran, M. Richard, R. André, and Le Si Dang, Phys. Rev. B 80, 045317 (2009).

[8] N. Y. Kim, K. Kusudo, C. Wu, N. Masumoto, A. Löffler, S. Höfling, N. Kumada, L. Worschech, A. Forchel, and Y. Yamamoto, Nature Phys 7, 681 (2011).

[9] M. Wouters, Phys. Rev. B 77, 121302 (2008).

[10] I. L. Aleiner, B. L. Altshuler and Y. G. Rubo, Phys. Rev. B 85, 121301 (2012).

[11] D. Sarchi, I. Carusotto, M. Wouters, and V. Savona, Phys. Rev. B 77, 125324 (2008).

[12] I. A. Shelykh, D. D. Solnyshkov, G. Pavlovic, and G. Malpuech, Phys. Rev. B 78, 041302 (2008).

[13] K. G. Lagoudakis, B. Pietka, M. Wouters, R. André, and B. Deveaud-Plédran, Phys. Rev. Lett. 105, 120403 (2010).

[14] A. P. D. Love, D. N. Krizhanovskii, D. M. Whittaker, R. Bouchekioua, D. Sanvitto, S. Al Rizeiqi, R. Bradley, M. S. Skolnick, P. R. Eastham, R. André, and Le Si Dang, Phys. Rev. Lett. 101, 067404 (2008).

[15] Th. Udem, R. Holzwarth, T. W. Hänsch, Nature 416, 233 (2002).

[16] Steven T. Cundiff and Jun Ye, Rev. Mod. Phys. 75, 325 (2003).

[17] P. Del'Haye, A. Schliesser, O. Arcizet, T. Wilken, R. Holzwarth, T. J. Kippenberg, Nature 450, 1214 (2007).

[18] T. J. Kippenberg, R. Holzwarth, S. A. Diddams, Science 332, 555 (2007).

[19] Note that $\mathrm{F}^{ \pm}$solutions are limit cycles in the full fourdimensional space $\left\{\psi_{1}, \psi_{2}\right\}$, which becomes essential in the presence of noise.

[20] These new solutions are limiting two-dimensional tori in the full four-dimensional space $\left\{\psi_{1}, \psi_{2}\right\}$, which will become particularly important in the presence of noise.

[21] N. V. Alexeeva, I. V. Barashenkov, K. Rayanov and S. Flach, Phys. Rev. A 89, 013848 (2014).

[22] Please see Supplemental Material at [URL will be inserted by publisher] for the derivation of the Lorentzian line width. 


\section{SUPPLEMENTAL MATERIAL}

This Supplemental Material presents the derivation of the noise-induced line properties of the $F^{ \pm}$limit cycles.

Let us rewrite the order parameters from (1) as

$$
\psi_{1,2}=\sqrt{n_{1,2}} e^{i \varphi_{1,2}} .
$$

From the equations of motion (2) it is straightforward to find the functions $A, B$ of the differential equations (we omit these details for brevity)

$$
\dot{n}_{1,2}=A_{1,2}\left(n_{1,2}, \varphi_{1,2}\right), \dot{\varphi}_{1,2}=B_{1,2}\left(n_{1,2}, \varphi_{1,2}\right) .
$$

Adding noise back to the equations of motion (2) yields

$$
\dot{\varphi}_{\mu}=B_{1,2}(t)+F_{\mu}(t), F_{\mu}=\frac{1}{2 i}\left(\frac{f_{\mu}}{\psi_{\mu}}-\frac{f_{\mu}^{*}}{\psi_{\mu}^{*}}\right) .
$$

The random process $F_{\mu}(t)$ is characterized by

$$
\left\langle F_{\mu}(t) F_{\mu}\left(t^{\prime}\right)\right\rangle=2 \kappa_{\mu}(t) \delta\left(t-t^{\prime}\right), \kappa_{\mu}=\frac{W}{4\left|\psi_{\mu}\right|^{2}} .
$$

In a $F^{ \pm}$limit cycle the noise-free evolution of $\varphi_{1,2}(t)$ is determined by the evolution of the total phase given by
$\dot{\Phi}=-\Omega_{0}$. The presence of noise gives

$$
\dot{\Phi}=-\Omega_{0}(t)+F(t), F(t)=\frac{1}{2}\left(F_{1}(t)+F_{2}(t)\right) .
$$

Since $\dot{n}_{1,2}=\dot{\varphi}=0$ we obtain

$$
\left\langle F(t) F\left(t^{\prime}\right)\right\rangle=2 \kappa \delta\left(t-t^{\prime}\right), \kappa=\frac{W}{16}\left[\frac{1}{n_{1}}+\frac{1}{n_{2}}\right] .
$$

The emission line is finally given by

$$
\begin{aligned}
I_{\mu}(\Omega) & =\frac{n_{\mu}}{\pi} \Re \int_{0}^{\infty} d t\left\langle e^{i \int_{0}^{t} F(\tau) d \tau} e^{i\left(\Omega-\Omega_{0}\right) t}\right\rangle \\
& =\frac{n_{\mu}}{\pi} \Re \int_{0}^{\infty} d t\left\langle e^{-\kappa t} e^{i\left(\Omega-\Omega_{0}\right) t}\right\rangle \\
& =\frac{n_{\mu}}{\pi} \frac{\kappa}{\kappa^{2}+\left(\Omega-\Omega_{0}\right)^{2}},
\end{aligned}
$$

which is a Lorentzian centred at $\Omega_{S}$ and of width $\kappa$. 